\title{
On the Nature of Radio Emission of AGNs: Spectra, Milli-arcsecond Structure and Polarization
}

\author{
Yu.A. Kovalev, Y.Y. Kovalev \\ Astro Space Center of the Lebedev Physical Institute, \\ Profsoyuznaya 84/32, Moscow, 117810 Russia
}

\begin{abstract}
The main observational properties of AGNs at centimeterdecimeter wavelengths - spectra, variability on various time scales, polarization and milli-arcsecond maps - are in agreement with calculations in a jet model with a strong quasi radial magnetic field. Typical spectra and VLBI structures can be explained by a simple case of a straight line jet, unlike the degree of the linear polarization. Curved jets in the model allow decreasing the polarization to observational values as well.
\end{abstract}

\section{Results}

We calculate and analyze synchrotron spectra, structure and polarization of a stationary and non-stationary continuous jet, ejected in a strong radial magnetic field from an active nucleus (magnetic pressure is much higher than jet pressure). An approach for the Hedgehog model, suggested by N.S.Kardashev in 1969 and discussed in details by Kovalev \& Kovalev (1997), is used.

The spectral shapes and the milli-arcsecond maps for stationary straight line jets agree with typical observations (Figure 1). Polarized structures have position angles transversal to the jet at the frequencies higher than the HF spectra maximum (similar to the Figure 1a, $P$ ), parallel to it - at the frequencies lower than the LF frequency twist, with both orientations in turn in two components - at the middle frequencies for the quasi-flat spectra region (similar to the Figure $1 \mathrm{~b}, P$, if the angular resolution is high enough), as expected.

Nevertheless, the linear polarization for the brightness in the model can be up to $70 \%$ in some regions - much more than it is usually observed by VLBI. This discrepancy can be explained by the following specific reasons in addition to known ordinary mechanisms of depolarization.

1. Observed polarized intensity maps strongly depend on the size, the shape and the orientation of the VLBI beam. This results in a decreasing of the polarization of visible intensity for a straight line stationary jet, if the angular resolution is not high along the jet.

2. A high polarized region located in the external optically thin part of a jet can be non-visible, if its brightness is much lower than that of the low polarized region. In the model it can occur in a wide frequency band.

3. Strong depolarization of visible intensity on the VLBI maps can occur, if a narrow jet is curved or its ejection is non-stationary. Moreover, total spectra can be the same as those of non-curved jets, for a special class of curvatures. 

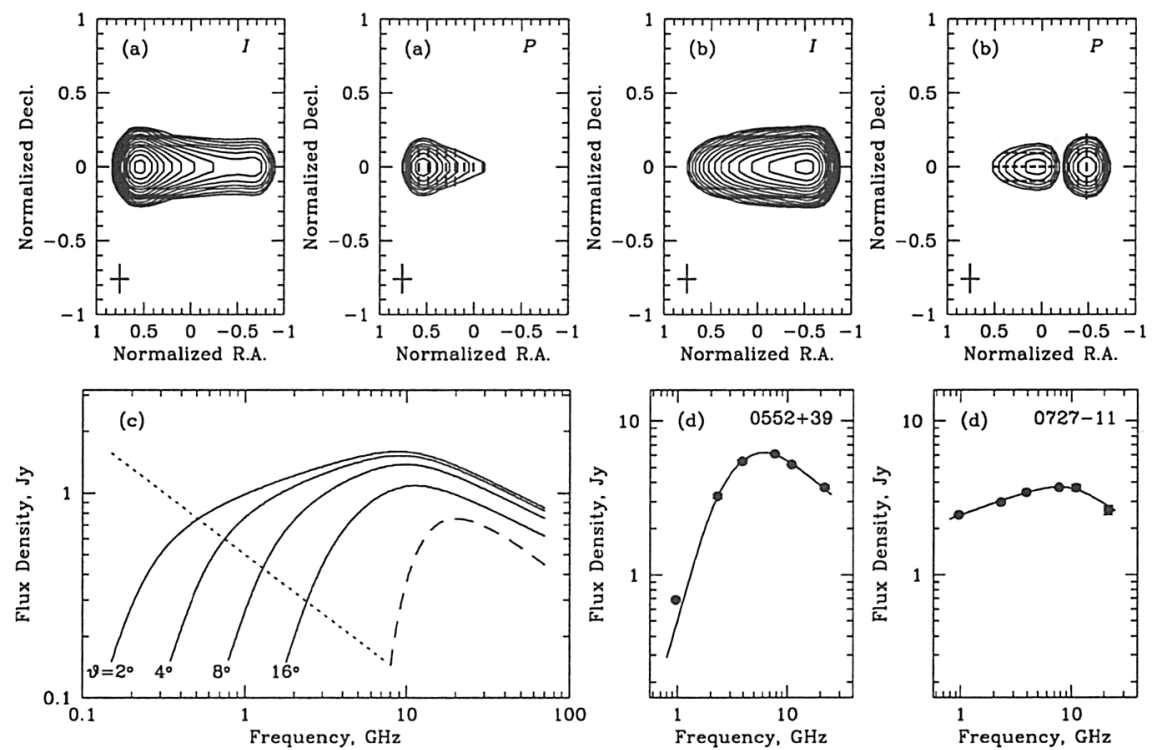

Figure 1. Some examples of the model analysis for a stationary jet: maps for total and polarized intensity at various frequencies (on top; beam used is shown by the cross) and spectra at various angles $\vartheta$ of the jet's axis to an observer line of sight (below). Maps (a) and (b) correspond to the frequencies higher and lower than the frequency of the maximum of spectra shown in (c), respectively. For a comparison we give the spectrum of a homogeneous synchrotron source (dashed lines). Total spectra (not shown) are often obtained by a sum of spectra with a spectrum of LF background component (dots). Fits for two AGNs from our 1-22 GHz observations (points) in 1998, March, at the RATAN-600, are presented in (d) by solid lines.

\section{Conclusions}

The jet model does not contradict the main observational properties of AGNs, including the existence and transitions between quasi-stationary and variable phases for spectra, structure and polarization (see e.g. simulation of the flare evolution in BL $0236+164$ in Kovalev \& Larionov, 1994).

Increasing the resolution angle (in Space VLBI or at $\mathrm{mm}$-wavelengths) can increase the polarization of the visible intensity of VLBI maps along jets, if the model is valid and if the curvature of jets is not too high.

Low values of integral polarization may indicate that many compact objects in the model can have curved jets with a non-stationary ejection.

\section{References}

Kovalev, Yu. A., \& Kovalev, Y. Y. 1997, Ap\&SS, 252, 133

Kovalev, Y. Y., \& Larionov, G. M. 1994, ALett., 20, 3 